\title{
Spectral classifications in the near infrared of stars with composite spectra
}

\section{Study of a sample of 137 objects with the Aurelie spectrograph ${ }^{\star}$}

\author{
N. Ginestet ${ }^{1}$, J.M. Carquillat ${ }^{1}$, and C. Jaschek ${ }^{2}$ \\ 1 Observatoire Midi-Pyrénées, UMR 5572 (CNRS), 14 avenue Edouard Belin, 31400 Toulouse, France \\ 2 Observatoire de Strasbourg, URA 1280 (CNRS), 11 rue de l'Université, 67000 Strasbourg, France
}

Received May 15; accepted July 3, 1998

\begin{abstract}
We provide spectral classifications for a sample of 137 stars mentioned as having composite spectra. The classifications were carried out on $33 \AA / \mathrm{mm}$ spectra in the region $8370-8870 \AA$. Of these 137 objects, 115 correspond in the infrared to cool stars $(\mathrm{G}, \mathrm{K}$ or $\mathrm{M})$ of luminosity classes III, II and I; for 22 stars, we find only hot spectra of types B, A, F or Am, so that they do not fulfil our definition of composite spectra. We detect four new Am stars, and one Am star (HD 70826) turns out to be a composite spectrum object.

As in Paper II, the cool components of composite spectra show a strong concentration in the vicinity of G8III.
\end{abstract}

Key words: stars: fundamental parameters - stars: infrared — binaries: spectroscopic

\section{Introduction}

The two preceding papers (Paper I: Ginestet et al. 1994; Paper II: Ginestet et al. 1997) provided:

- A study of MK standards in the region $8370-8780 \AA$, which established the validity in this region of the MK classification and provided the criteria which could be used. We showed also the interest of the near infrared spectral region for the classification of the cool companions of composite spectra (CS).

Let us remind briefly that in our studies we consider as composite spectra all those in which a hot dwarf (of spectral type $B$ or $A$ ) spectrum appears with that of a cool subgiant, giant or supergiant (of spectral types $G, K$ or $M)$.

Send offprint requests to: J.M. Carquillat

e-mail: Carquila@obs-mip.fr

* Based upon observations carried out at Observatoire de Haute-Provence (OHP).
- An application of our criteria to a sample of 180 known or suspected to be CS: 60 stars present a hot spectrum of spectral types B, A, Am or F in the near infrared, whereas 120 present a cool spectrum of spectral type G, $\mathrm{K}$ or $\mathrm{M}$.

These two papers are based on $33 \AA / \mathrm{mm}$ spectra obtained with the CARELEC spectrograph mounted on the $193 \mathrm{~cm}$ telescope of the OHP. We call attention to the fact that this instrument was used also to establish an Atlas of MK standards in the near infrared (Carquillat et al. 1997).

Most unfortunately in 1995 we encountered difficulties, since the spectrograph became reserved for extragalactic work and we had to change to the spectrograph AURELIE, mounted on the $152 \mathrm{~cm}$ telescope. Such a change had various implications for our work:

- we had to make a new calibration of MK standards and to reobserve a certain number of stars already classified to verify the joint with the previous system

- to prolongate the exposure times, with the resulting loss of limiting magnitude. As an advantage we have now a larger spectral region accessible, which includes the lines MgI 8806 and FeI 8824.

In the present paper we establish the spectral classifications of 137 objects from the near infrared, observed with the AURELIE spectrograph.

Let us mention in passing that numerous studies have been devoted in the last years to the near infrared region, from both the stellar and the extragalactic point of view. The interested reader will find the majority of these papers quoted in our Papers I and II, so that there is no need to repeat them here. 


\section{Samples, observations, calibrations and classification criteria}

\section{- Samples}

We have studied the behavior of $57 \mathrm{MK}$ standards and of 137 stars signaled as having composite spectra, on the basis of equivalent widths.

\section{- Composite spectra}

The sample of CS stars was selected, as for Paper II, from the list of Hynek (1938); some additional objects were taken from Markowitz (1969); Cowley $(1973,1976)$, Hoffleit \& Jaschek (1982) and Stickland (1988).

We did observe 86 stars designed as or suspected to be CS; we have also reobserved 51 CS already taken with CARELEC, both to ensure a perfect fit and to supplement the material for some stars of Paper II which present problems.

- MK standards

We observed 57 standards stars; 40 of these had already been studied in Paper I and were reobserved to permit the calibration of AURELIE with respect to CARELEC. Seventeen new stars were included to study the lines MgI 8806 and FeI 8824. This complete sample is representative of stars of spectral types $\mathrm{G}, \mathrm{K}$ and $\mathrm{M}$ and luminosity classes I, II, III and V.

The observational limits of our samples are $V<9.5$ and $\delta>-25^{\circ}$.

\section{- Observations}

The observations were made at the $152 \mathrm{~cm}$ telescope with the AURELIE spectrograph (Gillet et al. 1994) at a dispersion of $33 \AA / \mathrm{mm}$. Five observational runs were made in 1995 and 1996 and permitted to secure 194 objects (standards plus CS), usually with two spectra for each star.

At this dispersion one has access to $880 \AA$, instead of the $400 \AA$ available at the CARELEC spectrograph. We have used a region of $500 \AA$ extending between 8370 and $8870 \AA$, because for $\lambda<8370 \AA$ the spectra are dominated by strong telluric lines. The receiver for the AURELIE spectrograph is a Thomson double bar (TH 7832) constituted by two lines of 2048 photodiodes of $750 \times 13 \mu \mathrm{m}$, each line (even and uneven pixels) being read by two lateral CCD's.

The treatment of spectra is here definitely simplified respect to CARELEC, since we have to deal only with unidimensional images. As with CARELEC a tungsten lamp has been used for flat field, and a neon spectrum for calibrations in wavelength. The normalisation of the spectra and the measurement of the equivalent widths has been made in a similar way to those made with CARELEC (see Paper I) with the help of the IHAP software available at the OHP.

\section{- Calibrations CARELEC/AURELIE}

To calibrate the spectra obtained with Aurelie with regard to those obtained with Carelec, we have measured the equivalent widths of some blends and lines selected over the length of the spectrum for the $40 \mathrm{MK}$ standards. We have chosen the blend $8468 \AA$, the CaII triplet (8498, $8542,8662 \AA)$, FeI $8688 \AA$ and the total absorption between 8390 and $8775 \AA$ (TA).

The measurements carried out with the two spectrographs are given in Figs. 1-4; on these graphs we have also plotted the equivalent width's of the CS measured.

We find that for the shorter wavelengths (blend 8468 and CaII triplet) the equivalent widths obtained with Aurelie are, in the mean, weaker than those measured with Carelec, whereas for the longer wavelengths (FeI 8688) and for the total absorption TA there is no significant difference.

A careful comparison of the spectra show effectively that the lines observed with Aurelie are less deep than when observed with Carelec. This translates into a correction of the sum of the three equivalent widths of the CaII lines by an average correction of $0.5 \AA$, i.e. a 3 to $7 \%$ correction of the equivalent width. On the other side, the continuous background of the spectrum is sligthly inferior to the pseudocontinuum at the longer wavelengths (essentially for $\lambda>8662 \AA$ ) because we had to adjust it over a longer wavelength range than for the Carelec spectra and these two effects seem to compensate for TA and FeI 8688.

We still compare in Sect. 3 the classifications made with material from the two spectrographs to see if with all corrections made there remains still a systematic effect.

\section{- Classification criteria}

The classification of the 115 objects which present a cool stellar spectrum ( $\mathrm{G}, \mathrm{K}$ or $\mathrm{M})$ in the near infrared has been carried out using the same criteria defined in Paper I and using the same technique as described in Paper II. But since the zone observable with Aurelie is larger than the zone observable with Carelec, we have been able to study with the help of the $57 \mathrm{MK}$ standards the behavior of two supplementary intense lines, MgI 8806 and FeI 8824.

We have found in Paper I that a relation exists between the spectral type and the ratio of the central depths $(R)$ of the neighbouring lines TiI 8683 and FeI 8679.

The mean relation was as follows:

$\begin{array}{lrrrrrrr}\text { Spectral Type ............ } & \text { G0 } & \text { G2 } & \text { G5 } & \text { G8 } & \text { K0 } & \text { K3 } & \text { K5 } \\ R \text { (classes V, III, II) } \ldots . . & 0.32 & 0.40 & 0.50 & 0.70 & 0.85 & 1.30 & 1.85 \\ R \text { (classe Ib) } \ldots \ldots \ldots \ldots \ldots \ldots . . & 0.40 & 0.52 & 0.80 & 1.06 & 1.26 & 1.60 & 1.85 .\end{array}$

We have therefore used this ratio $R$ instead of the spectral type (assumed unknown for the CS) for the study of the behaviour of the lines MgI 8806 and FeI 8824: both lines present a positive luminosity effect (see Fig. 2). We have also considered the ratio of the equivalent widths of MgI 8806 and FeI 8688, as well as the ratio MgI 8806/FeI 8824. These two relations present a negative luminosity effect and permit a separation of giants and supergiants (Fig. 3). Dwarfs figure also on these graphs, in spite of their small number; they seem to behave like giants. 

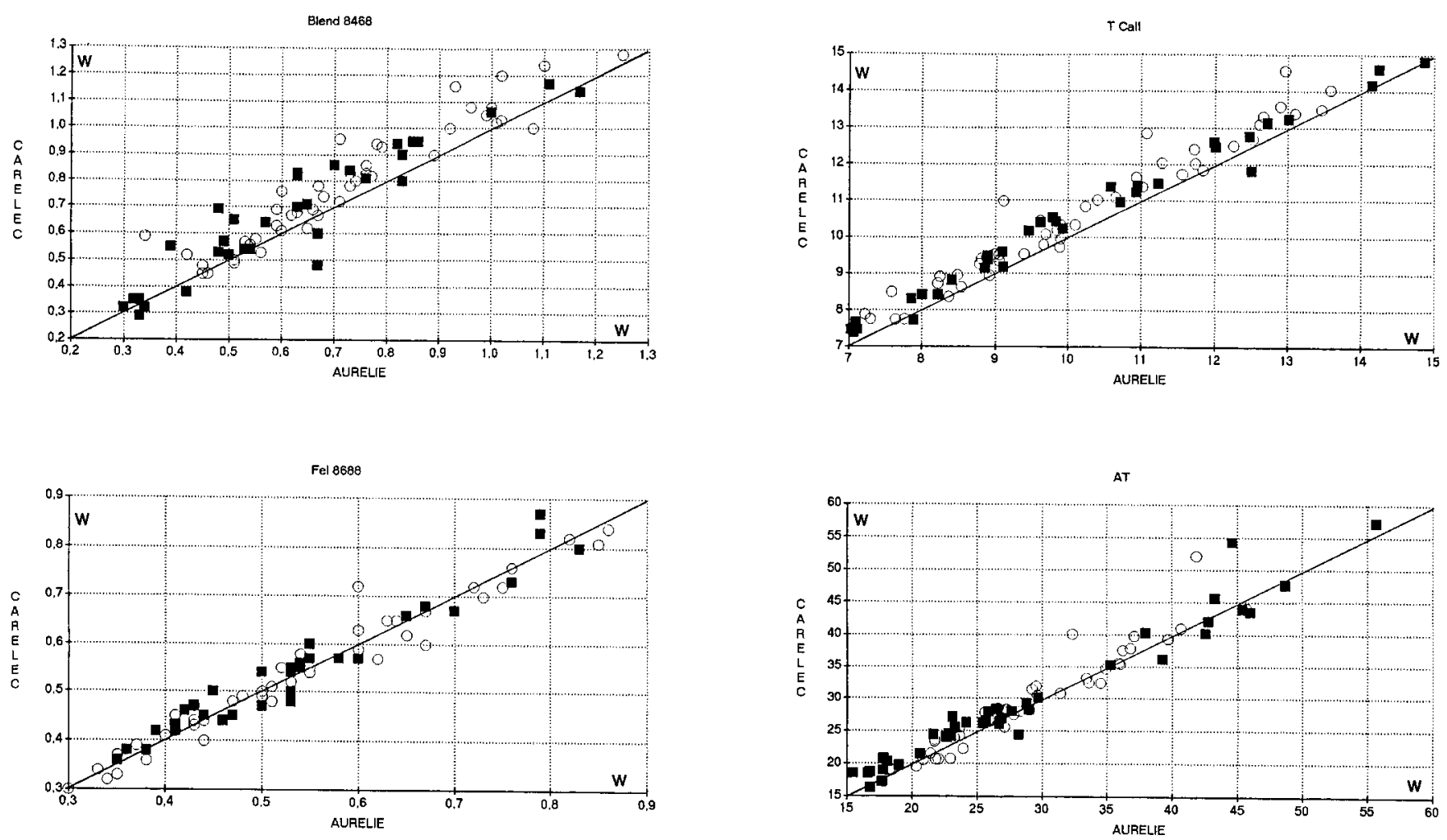

Fig. 1. Comparison of equivalent widths measured from CARELEC and AURELIE spectrographs for the $8468 \AA$ blend, the Triplet of CaII, the FeI 8688 line and the Total Absorption between $8390 \AA$ and $8775 \AA$. Filled squares: MK standards; open circles: composite spectra
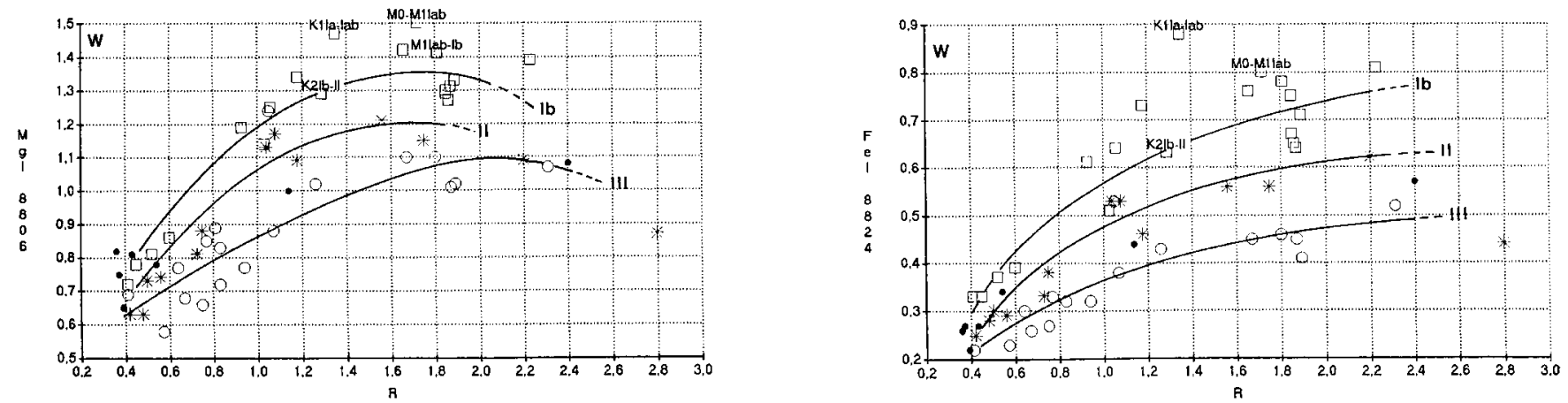

Fig. 2. Behaviour of the equivalent widths of the $\mathrm{MgI} 8806$ and FeI 8824 lines versus $R$ (correlated with the spectral type, see text) for MK standards. Open squares: supergiants; asterisks: bright giants; open circles: giants; dots: dwarfs

\section{Results and discussion}

As said before we have observed 137 stars designated as CS or candidates. A first examination permits to separate 22 stars which have a spectrum of a hot star of type B, $\mathrm{A}, \mathrm{Am}$ or $\mathrm{F}$ in the near infrared. These stars are listed in Table 1. The other 115 stars have a cool spectrum of types G, K or M. These are listed in Table 2, except 20 stars for which the classification from Aurelie and from Carelec coincide totally (see part 3a). In the two tables the data $V$ and $B-V$ were taken from the Hipparcos and
Tycho catalogues (1997). If they are not contained in these catalogues, the data come from the Centre de Données Stellaires (CDS) or from Hynek (1938). An asterisk implies a note at the end of the table. In the column "references", for reasons of space economy, figures only the name of the first author of the paper.

We next will validate our classifications obtained from Aurelie with proceeding from Carelec. Then we shall confront our classifications with those of the "standard" CS, that is, stars which possess a classification which we consider trustworthy (see Paper II, 4.1). And finally we shall 

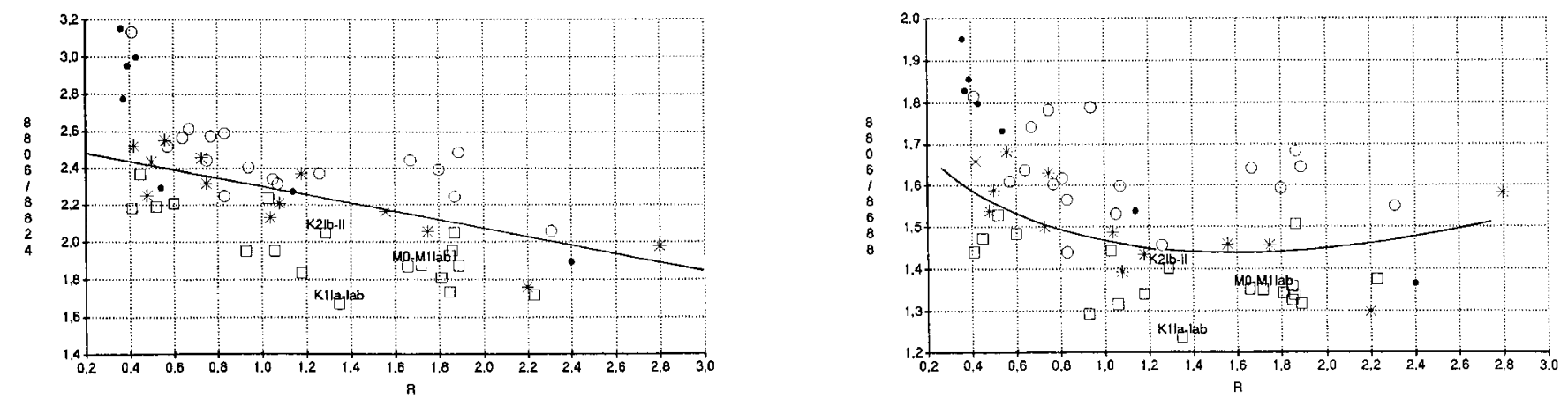

Fig. 3. Behaviour of the MgI 8806/FeI 8824 and MgI 8806/FeI 8688 equivalent width ratios versus $R$ (correlated with the spectral type, see text) for MK standards. Open squares: supergiants; asterisks: bright giants; open circles: giants; dots: dwarfs
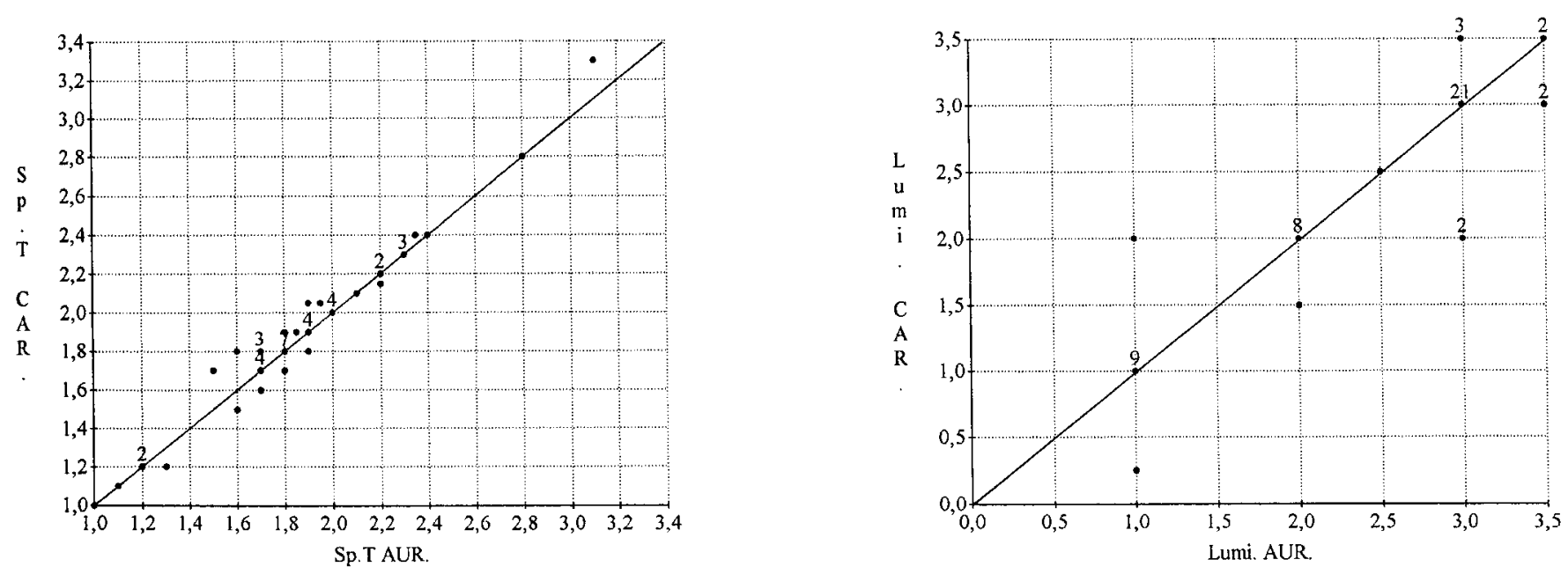

Fig. 4. Comparison of the Spectral Types (Sp T) and Luminosity Classes (Lumi.) for 51 composite spectra classified with both the CARELEC and AURELIE spectrographs. Sp T: G...1, K...2, M...3; Lumi.: Ia...0, Ib...1, II...2, III...3; numbers of the plots are weights

confront, for a small sample, our results with those published in the Michigan catalogues (Houk 1982; Houk \& Smith-Moore 1988).

\section{a) Comparison Carelec-Aurelie}

A comparison of the 51 stars observed with both spectrographs permits to isolate 22 stars for which the results are identical. These are: HD 4775 (G7III) - 17878 (G7III) - 21771 (K3II) - 23089 (G2Ib) - 25555 (K1II) 26630 (G0Ib) - 26673 (G9II) - 29094 (G7Ib) - 39118 (K0II) - 66094 (G9III-IV) - 74395 (G1Ib) - 88021 (G8III) - 166479 (G8III) - 169985 (G9III) - 184759 (G8III) - 187259 (G8III) - 193410 (K0III) - 193495 (G9II) - 197177 (G8II) - 200428 (K0III) - 205114 (G2Ib) 213310 (K6-M0I). These objects do not figure in Table 2, except HD 4775 and HD 25555 for which recent classifications exist, obtained on the basis of the method of substraction of spectra (Griffin R\&R 1986). For the other 29 stars one perceives small differences in spectral type and luminosity classes (Fig. 4), but without a systematic trend. The average scatter around the linear relations corresponds to about 0.7 tenth of a spectral type and 0.3 lu- minosity class. Both errors are within the normal precision of spectral types (Jaschek \& Jaschek 1987).

b) Comparison with the so called "standards" CS

We have made the inventory of CS stars for which a satisfactory classification exists (see Paper II). The complete list is as follows: HD 4775, 17878, 25555, 26630, 29094, 57146, 74395, 88021, 169689, 173764, 184759, 187076, 187259, 187299, 190161, 192713, 200428, 200905, 201270, 205114.

Of this list 13 have classifications obtained by $R \& R$ Griffin by the so called method of substraction of spectra and six others are MK standards. In Fig. 5 we compare our Aurelie classifications with those of the "standards" for spectral types and for luminosity classes respectively. From the dispersions around the average relation we find again values which are normal for the errors of classification (one tenth of spectral type and 0.25 luminosity class). Also there does not exist a systematic effect.

c) Comparison with the classification of the Michigan catalogues

Our complete sample (Papers II and III) contains 25 
Table 1. Stars of the sample which exhibit a hot spectrum

\begin{tabular}{|c|c|c|c|c|c|c|c|}
\hline $\mathrm{HD} / \mathrm{BD}$ & $\mathbf{A 2 0 0 0}$ & D2000 & $\bar{V}$ & $B-V$ & $\overline{\mathbf{S P}}$ & Références & SP (IR) \\
\hline 1952 & $\begin{array}{ccc}h & m & s \\
00 & 24 & 05.2\end{array}$ & $\begin{array}{r}\circ \prime \prime \prime \\
+441553\end{array}$ & 6.66 & 0.413 & \begin{tabular}{|l} 
F6III+A4V \\
F3III \\
F5V \\
\end{tabular} & $\begin{array}{l}\text { Markowitz (1969) } \\
\text { Bideiman (1988) } \\
\text { Sato (1990) }\end{array}$ & F2III \\
\hline 5621 & $0103 \quad 37.7$ & +843626 & 6.74 & 0.246 & $\begin{array}{l}\text { F5p } \\
\text { A8V+G0II-III }\end{array}$ & $\begin{array}{l}\text { Cat. HD } \\
\text { Markowitz (1969) }\end{array}$ & $\overline{\text { A8IV }}$ \\
\hline 13728 & $\begin{array}{llll}02 & 13 & 42.1\end{array}$ & -030154 & 7.33 & 0.338 & $\begin{array}{l}\mathrm{Am} \\
\mathrm{Am}\end{array}$ & $\begin{array}{l}\text { Olsen (1979) } \\
\text { Griffin (1993) } \\
\end{array}$ & $\mathbf{A m}$ \\
\hline 29354 & $\begin{array}{l}0437 \quad 37.3 \\
\end{array}$ & +045858 & 7.62 & 0.344 & Fo & Cat. HD & F0IV \\
\hline 39569 & $\begin{array}{lll}04 & 54 & 12.1 \\
\end{array}$ & +162040 & 8.20 & & $\mathrm{A3}$ & Cat. HD & Fiv \\
\hline 60092 & \begin{tabular}{|l|l|l|}
07 & 32 & 16.3 \\
\end{tabular} & $-1831 \quad 03$ & 7.34 & 0.411 & Fm delta Del & Houk (1988) & $\mathbf{A m}$ \\
\hline 70318 & $\begin{array}{llll}08 & 2153.9 \\
\end{array}$ & +173605 & 8.23 & 0.349 & $\mathrm{A3}$ & Cat. HD & $\mathbf{A m}$ \\
\hline 97336 & $11 \quad 12 \quad 30.1$ & +243906 & 8.15 & 0.357 & F5A5 & Cat. HD & F5wl \\
\hline 103885 & 115760.0 & +734654 & 8.80 & 0.445 & F2 & Cat. HD & F5V \\
\hline$\overline{108283}$ & 122624.0 & +271606 & 4.92 & 0.277 & $\begin{array}{l}\text { F0IIInp } \\
\text { F0III sh } \\
\text { A9 sh } \\
\text { A9 Sr } \\
\text { A9Vp lamb. Boo }\end{array}$ & $\begin{array}{l}\text { Cowley (1969) } \\
\text { Slettebak (1982) } \\
\text { Jaschek (1991) } \\
\text { Malanushenko (1994) } \\
\text { Abt (1995) }\end{array}$ & A9p \\
\hline $111844-5$ & 125154.6 & +191005 & 7.29 & 0.569 & $\begin{array}{l}\text { Am; F8-G0III } \\
\text { A8IVp; F8IV } \\
\text { Am; F8V } \\
\end{array}$ & $\begin{array}{l}\text { Berger (1962) } \\
\text { Cowley (1967) } \\
\text { Abt (1981) }\end{array}$ & Am ; F9III- * \\
\hline$\overline{121800}$ & $\begin{array}{lll}13 & 55 & 15.4\end{array}$ & +660700 & 9.04 & -0.093 & $\begin{array}{l}\mathrm{Gp} \\
\mathrm{B} 1,5 \mathrm{~V} \\
\mathrm{G} 7\end{array}$ & $\begin{array}{l}\text { Cat. HD } \\
\text { Dworetsky (1982) } \\
\text { CDS }\end{array}$ & B shell * \\
\hline 135631 & $\begin{array}{llll}15 & 14 & 56.7\end{array}$ & $+38 \quad 18 \quad 02$ & 7.11 & 0.359 & $\begin{array}{l}\text { Fop } \\
\text { FIV }\end{array}$ & $\begin{array}{l}\text { Cat. HD } \\
\text { Abt (1981) }\end{array}$ & $\overline{\text { FOV }}$ \\
\hline 140283 & $15 \quad 4307.2$ & -105545 & 7.20 & 0.484 & $\begin{array}{l}\text { A8V var. } \\
\text { F3VI } \\
\text { G0IV-V m-5 }\end{array}$ & $\begin{array}{l}\text { Keenan (1953) } \\
\text { Evans (1966) } \\
\text { Gray (1989) } \\
\end{array}$ & F9w1 \\
\hline 141652 & 153939.2 & +795858 & 6.89 & 0.392 & F2V & Abt (1981) & FIV \\
\hline 142846 & $15 \quad 56 \quad 14.9$ & +144504 & 8.28 & 0.400 & F2IV & Bidelman (1951) & F2V \\
\hline 147275 & $\begin{array}{lll}16 & 19 & 14.5 \\
\end{array}$ & +413932 & 8.01 & 0.363 & A5G & Cat. HD & FOV * \\
\hline 154225 & 170221.0 & +400425 & 7.99 & 0.439 & A5 & Cat. $\mathrm{HD}$ & $\mathbf{A m}$ \\
\hline 224738 & $\begin{array}{lllll}00 & 00 & 18.4 \\
\end{array}$ & +655641 & 8.50 & & $\mathrm{~F}$ & Cat. $\mathrm{HD}$ & $\mathbf{A m}$ \\
\hline 232625 & $\begin{array}{llll}02 & 16 & 15.0\end{array}$ & $+52 \quad 1724$ & 9.4 & & $\begin{array}{l}\mathrm{F} \\
\mathrm{F} 5\end{array}$ & $\begin{array}{l}\text { Cat. HD } \\
\text { CDS }\end{array}$ & $\overline{\text { F4V }}$ \\
\hline $232767-8$ & $03 \quad 15 \quad 30.4$ & +513105 & 9.74 & 0.633 & $\mathrm{~K} 5 \mathrm{~A}$ & Cat. $\mathrm{HD}$ & Am \\
\hline 234729-30 & $\begin{array}{llll}1851 & 39.1 \\
\end{array}$ & $+51 \quad 1550$ & 9.28 & 1.000 & G5A0 & Cat. HD & F3III: \\
\hline
\end{tabular}

Notes:

.HD 111844-5: ADS 8690; the spectra of the components are obtained separately (Sep. 16").

HD 121800: one spectrum in the blue region leads to B2 shell.

. HD 147275: could be a marginal Am.
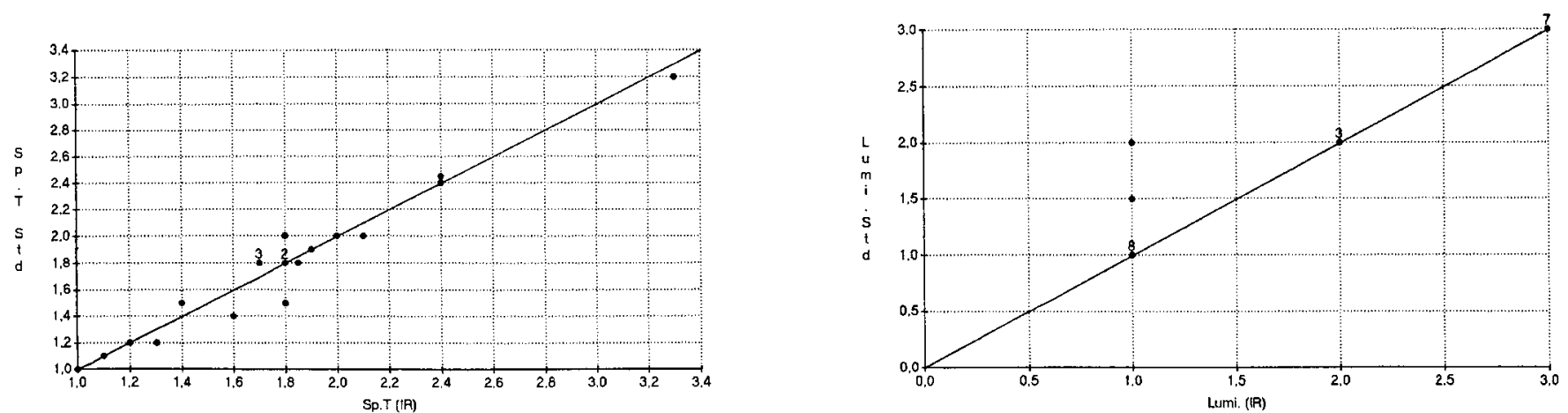

Fig. 5. Comparison of infrared MK classifications (spectral types and luminosity classes) with those of "standard composite spectra" (see text). Sp T: G...1, K...2, M...3; Lumi.: Ia...0, Ib...1, II...2, III...3; numbers on the plots are weights 
Table 2. Classifications of the cool components, SP(IR), of stars with composites spectra

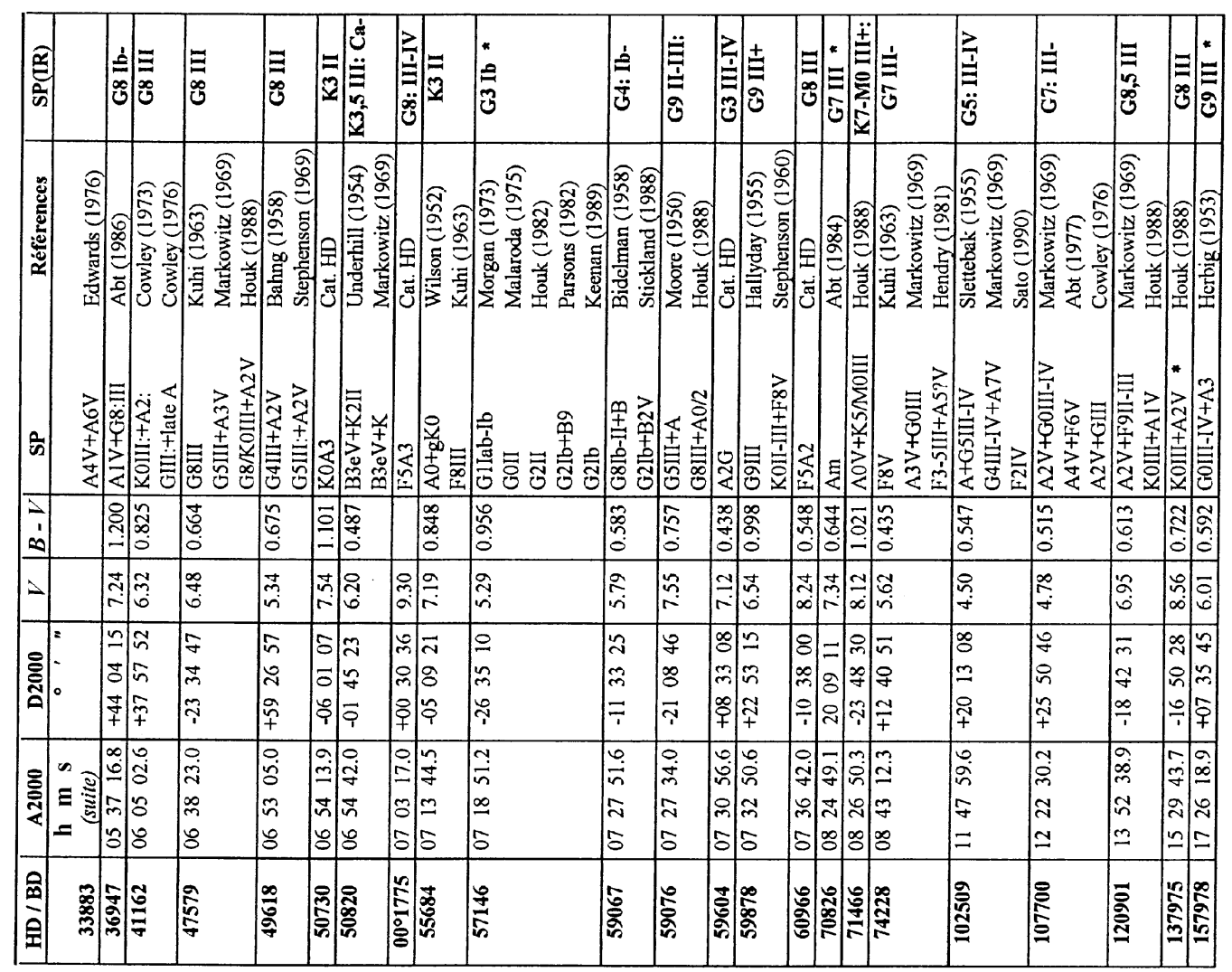

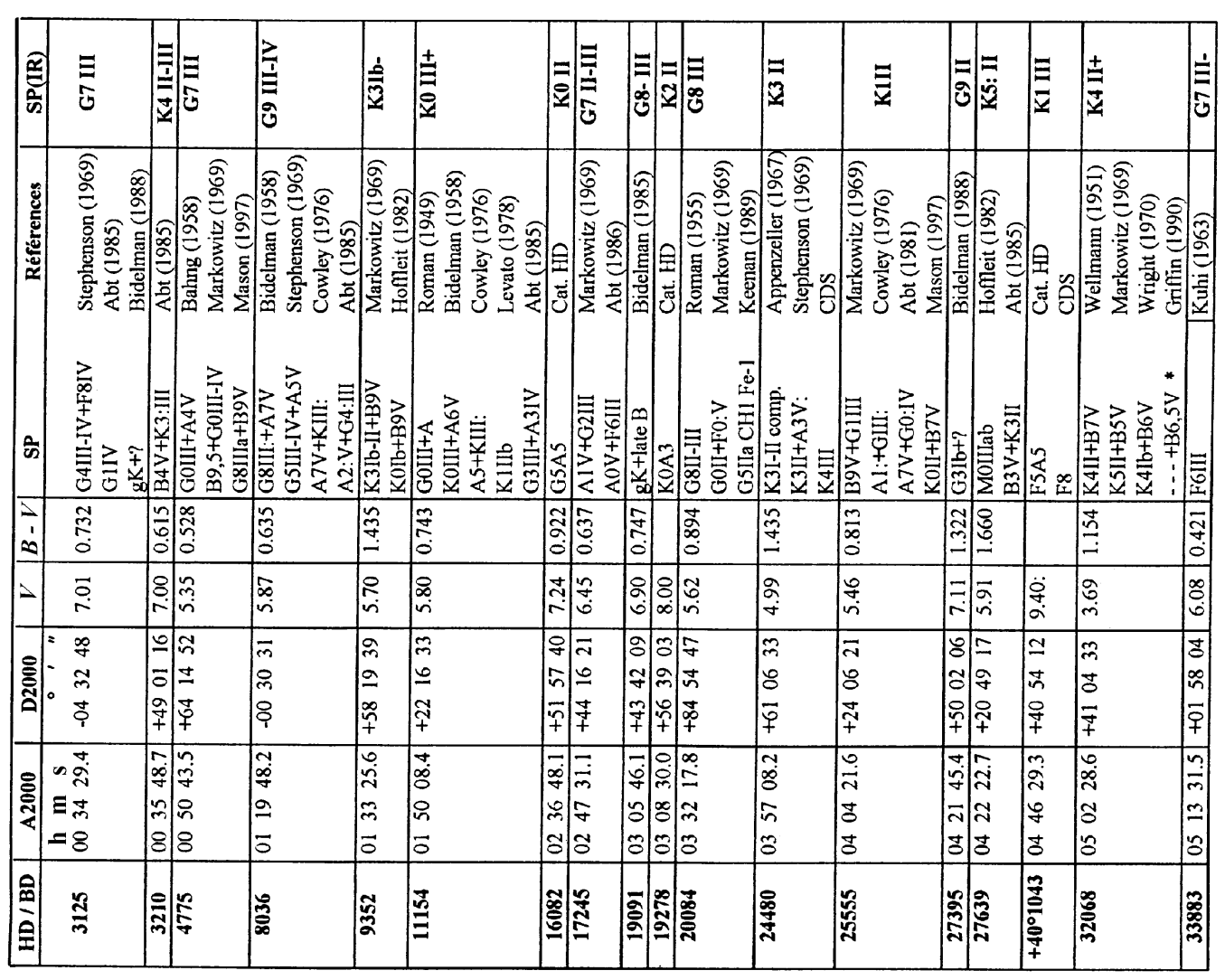


Table 2. continued

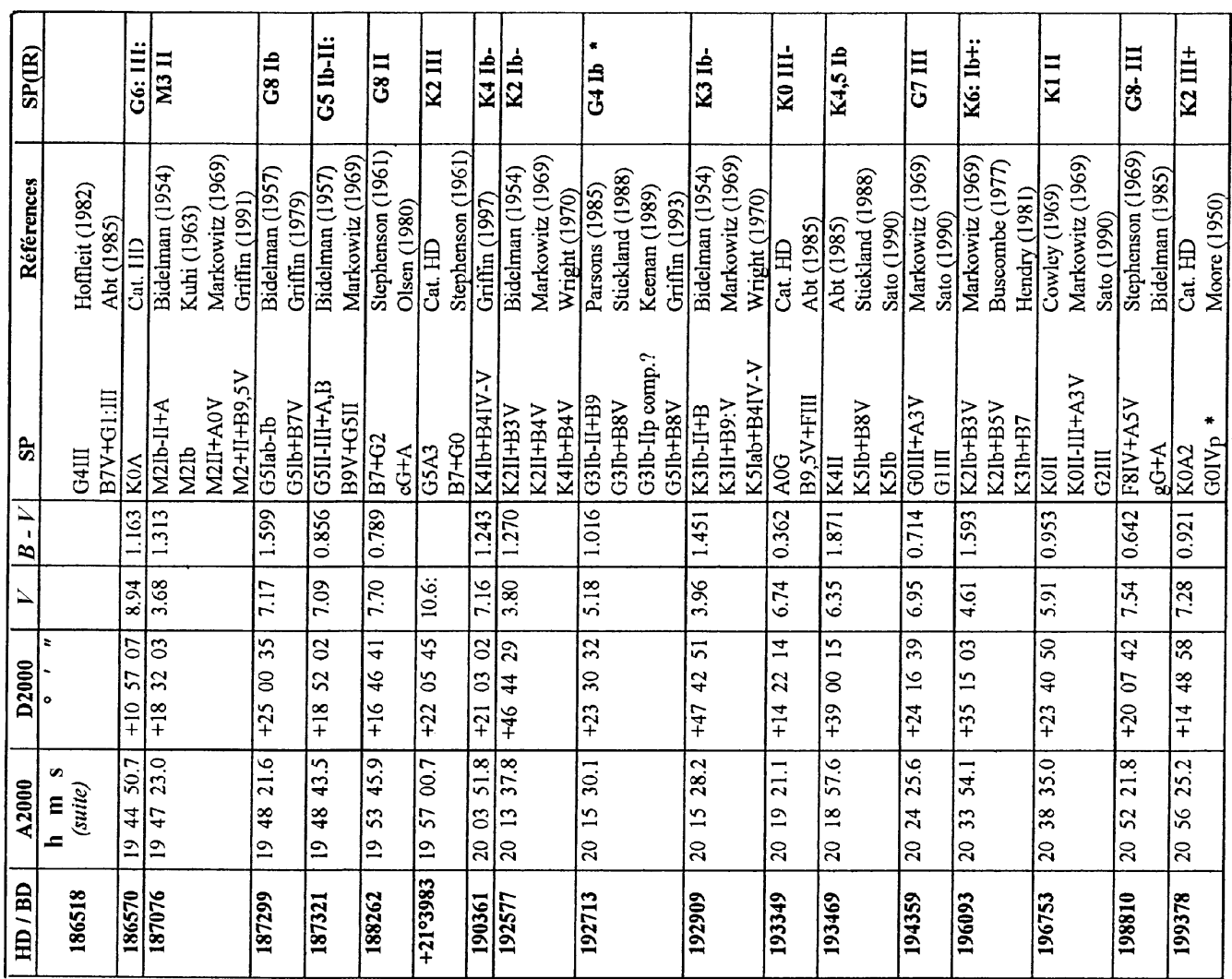

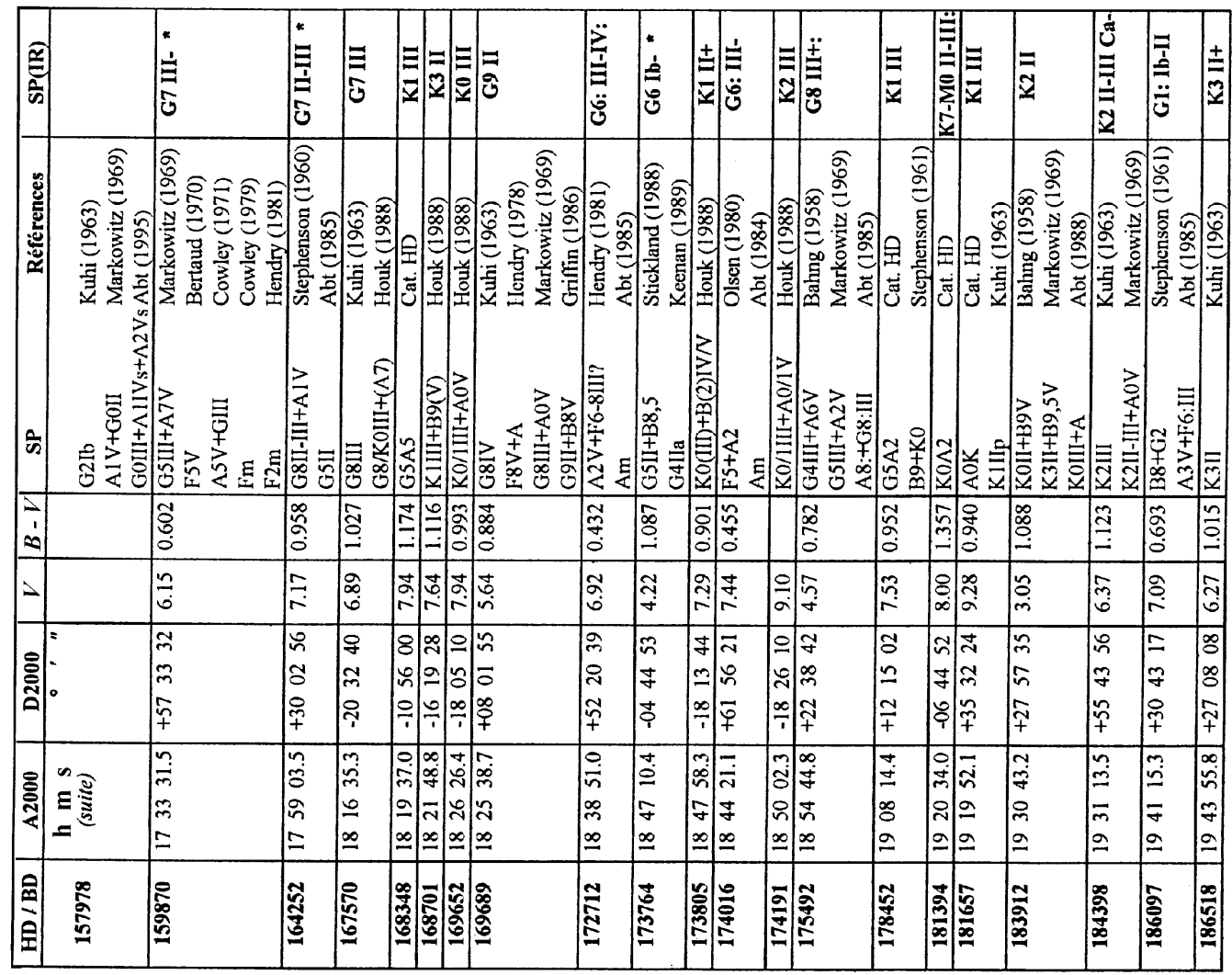


Table 2. continued

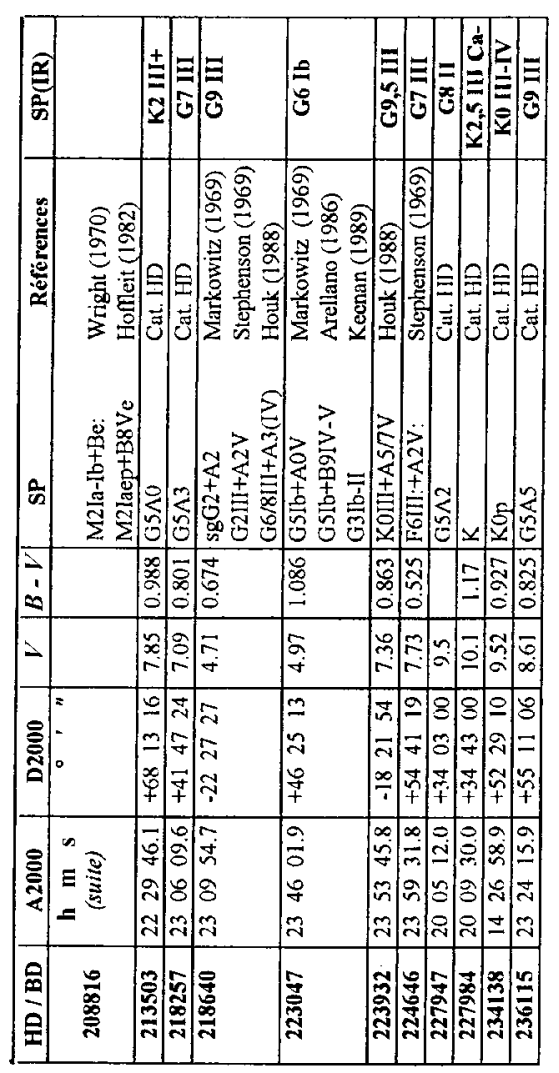

\begin{tabular}{|c|c|c|c|c|c|c|c|}
\hline$\frac{\hat{\alpha}}{\hat{\nu}}$ & $\begin{array}{l}: \\
\dot{*} \\
\ddot{\Sigma}\end{array}$ & $\mid \begin{array}{l}\equiv \\
\sqrt[3]{2} \\
0 \\
5\end{array}$ & 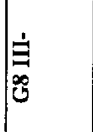 & $\bar{\Sigma}$ & 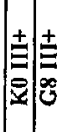 & 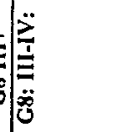 & $\ddot{z}$ \\
\hline 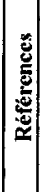 & 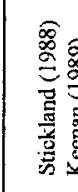 & 象象 & 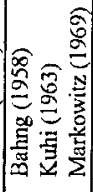 & 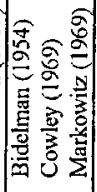 & 重 & 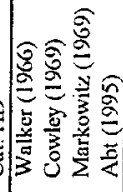 & a \\
\hline $\mid \frac{4}{2}$ & 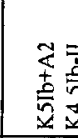 & : & 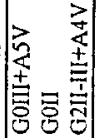 & 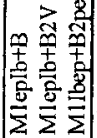 & $\sqrt{0}$ & 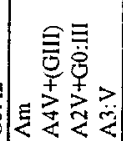 & 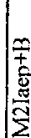 \\
\hline $\begin{array}{l}3 \\
0\end{array}$ & 参 & 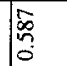 & 高 & $\Xi$ & 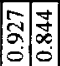 & $\mid \begin{array}{c}\infty \\
\substack{\infty \\
0}\end{array}$ & 1 \\
\hline$=$ & $\underset{\sim}{\stackrel{\sim}{\pi}}$ & $\underset{\sim}{\curvearrowright}$ & $\underset{m}{\alpha}$ & ज्ञ & 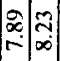 & {$\left[\begin{array}{c}n \\
n \\
0\end{array}\right.$} & $\bar{n}$ \\
\hline 㟃 & $\begin{array}{l}= \\
= \\
- \\
-5 \\
0 \\
0 \\
0 \\
7 \\
7\end{array}$ & $\begin{array}{l}m \\
m \\
q \\
a \\
q\end{array}$ & $\mid \begin{array}{l}\tilde{n} \\
\dot{y} \\
n \\
\vdots\end{array}$ & 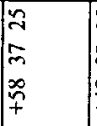 &  & $\mid \begin{array}{l}\infty \\
0 \\
3 \\
3 \\
7\end{array}$ & $m$ \\
\hline & $\begin{array}{l}\tilde{n} \\
\tilde{n} \\
=0 \\
=\bar{n}\end{array}$ & $\begin{array}{l}\vec{i} \\
\tilde{n} \\
8 \\
\bar{n} \\
\vec{n}\end{array}$ & $\begin{array}{l}m \\
a \\
a \\
n \\
\vec{n}\end{array}$ & $\begin{array}{l}0 \\
i \\
a \\
a \\
a\end{array}$ & 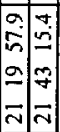 & 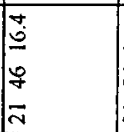 & $\mid \begin{array}{l}\bar{a} \\
m \\
\infty \\
\bar{n} \\
\bar{N}\end{array}$ \\
\hline 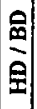 & 訚 & 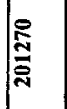 & 售 & 㒀 & 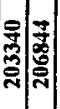 & 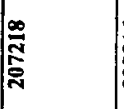 & \\
\hline
\end{tabular}

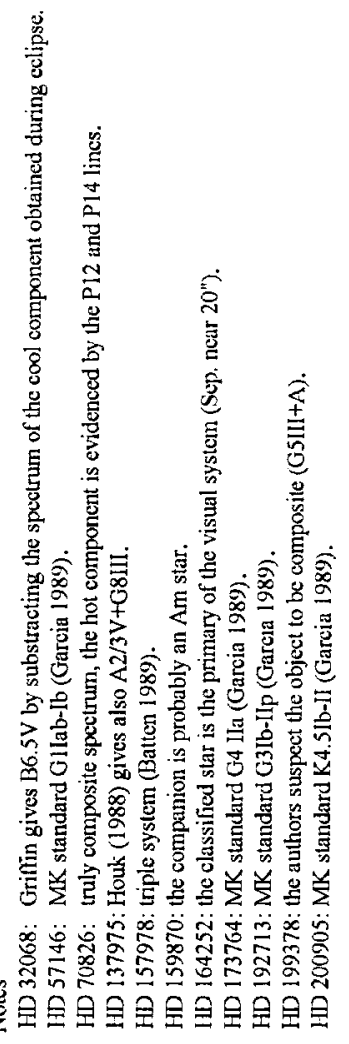



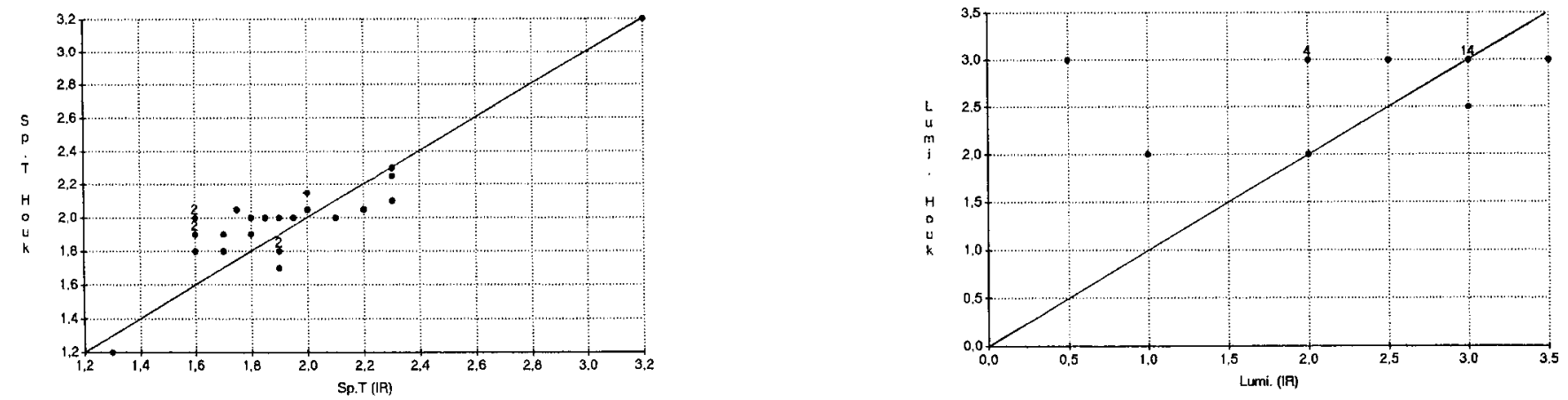

Fig. 6. Comparison of infrared MK classifications (spectral types and luminosity classes) with those of N. Houk (Michigan Catalogues). Sp T: G...1, K...2, M...3; Lumi.: Ia...0, Ib...1, II...2, III...3; numbers on the plots are weights

stars of the southern hemisphere which figure in the catalogues of Michigan: HD 29961, 35162, 47579, 51250, 57146, $59076,60414,70442,74946,75098,79267,84367,95235$, $102171,120901,137975,167570,168701,169652,173805$ 174191, 193495, 209278, 218640, 223932.

In Fig. 6 we compare their and our classifications, for spectral types and luminosity classes respectively. We notice that for HD 209278 Houk does not provide the luminosity classification.

The spectra classed by Houk were obtained with the objective prism, on IIaO plates, dispersion $108 \AA / \mathrm{mm}$ at $\mathrm{H}_{\gamma}$ and a resolution near to $2 \AA$. If one takes into account the difficulties in classifying CS in this wavelength region (see Paper I), the agreement with our classifications is very good for the spectral types. On the average, the Michigan classifications are slightly later than ours for type $\mathrm{G}$ and slightly earlier for type K. For luminosity classes the agreement is excellent for class III, but in disagreement for classes I and II. From five stars classed II by us, four are classed III by Houk and two stars classed I by us are classed II and III by Houk.

\section{Conclusions}

Of the 86 stars classed for the first time in the near infrared, 27 had not MK classification to our knowledge. Of these 27, 4 are Am, 7 are F type classes V or IV, 11 are cool giants and 5 bright giants.

Let us also point out an example of confusion between Am and CS. Usually one finds Am stars called CS, but here we find an Am which is CS. The object is HD 70826 which figures as Am in Hauck (1986). We included it in our list of CS candidates because its correlation peak in the CORAVEL instrument for the measure of radial velocities did not correspond to an Am star.

We detect again, as Paper II, a large quantity of giants between types G7 and K2 with a maximum around G8 and an absence of early type $G$ giants which predominated in the old classifications.
Our program of classification of cool components of CS stars is now complete (Papers II and III). From 266 stars observed as true CS or candidates, we found in the near infrared 184 spectral types of cool stars ( $G, K$ and $M)$ and 82 corresponding to early type stars (B, A, Am, F).

We hope to continue this study in two directions. On one hand we wish to classify the hotter components in the blue region, since there exist a wide disparity of classifications for the same object. This would enable us to carry out a sound statistical study of the group. We notice that the majority of the radial velocities of these binaries are being studied by R. Griffin (Cambridge Observatory) and by ourselves with CORAVEL (OHP), so that for a certain number of objects the orbital elements are already known. The other direction is to confront the whole set of our classifications with the data from the HipparcosTycho catalogues.

Acknowledgements. We appreciate very much the data and bibliographic references made available from the CDS at Strasbourg.

\section{References}

Abt H.A., Levato H., 1977, PASP 89, 29

Abt H.A., 1981, ApJS 45, 437

Abt H.A., 1984, ApJ 285, 247

Abt H.A., 1985, ApJS 59, 95

Abt H.A., 1986, ApJ 309, 260

Abt H.A., 1988, ApJ 331, 922

Abt H.A., Morrell N.I., 1995, ApJ 99, 135

Appenzeller I., 1967, PASP 79, 102

Arellano Ferro A., Madore B.F., 1986, ApJ 302, 767

Bahng J.D.R., 1958, ApJ 128, 572

Batten A.H., Fletcher JM., MacCarthy D.G., 1989, Publ. Dom. Astrophys. Obs. 17, 1

Berger J., 1962, Ann. Ap 25, 1

Bertaud Ch., 1970, A\&AS 1, 7

Bidelman W.P., 1951, ApJ 113, 304

Bidelman W.P., 1954, ApJS 1, 175

Bidelman W.P., 1957, PASP 69, 147, 326

Bidelman W.P., 1958, PASP 70, 168 
Bidelman W.P., 1985, AJ 90, 341

Bidelman W.P., 1988, PASP 100, 1084

Buscombe W.B., 1977, MK Spectral Classifications: Third General Catalogue. Northwestern Univ., Evanston

Carquillat J.M., Jaschek C., Jaschek M., Ginestet N., 1997, A\&AS 123, 5

Cowley A.P., Jaschek M., Jaschek C., Cowley R.C., 1967, PASP 79, 497

Cowley A.P., 1969, PASP 81, 297

Cowley A. \& C., Jaschek M. \& C., 1969, AJ 74, 375

Cowley A.P., Crawford D.L., 1971, PASP 83, 296

Cowley A., 1973, PASP 85, 314

Cowley A.P., 1976, PASP 88, 95

Cowley A.P., Bidelman W.P., 1979, PASP 91, 83

Dworetsky M.M., Whitelock P.A., Carnochan D.J., 1982, MNRAS 201, 901

Edwards T.W., 1976, AJ 81, 245

Evans D.S., 1966, Royal Obs. Bull. 110, 185

Garcia B., 1989, Bull. Inform. CDS 36, 27

Gillet D., Burnage R., Kohler D., et al., 1994, A\&AS 108, 181

Ginestet N., Carquillat J.M., Jaschek M., Jaschek C., 1994, A\&AS 108, 359

Ginestet N., Carquillat J.M., Jaschek M., Jaschek C., 1997, A\&AS 123, 135

Gray R.O., 1989, AJ 98, 1049

Griffin R. \& R., 1979, MNRAS 187, 91

Griffin R. \& R., 1986, JA\&A 7, 195

Griffin R. \& R., 1990, JA\&A 11, 281

Griffin R.E.M., Griffin R.F., Schröder K.-P., Reimers D., 1990, A\&A 234, 284

Griffin R.E.M., 1991, The Observatory 111, 248

Griffin R.E.M., Hünsch M., Marshall K.P., Griffin R.F., Schröder K.-P., 1993, A\&A 274, 225

Griffin R.F., 1993, The Observatory 113, 32

Griffin R. \& R., 1997, MNRAS 285, 472

Hallyday I., 1955, ApJ 122, 222

Hauck B., 1986, A\&AS 64, 21

Hendry E.M., 1978, AJ 83, 615

Hendry E.M., 1981, AJ 86, 271

Herbig G.H., Turner B.A., 1953, ApJ 118, 447

Hoffleit D., Jaschek C., 1982, The Bright Star Catalogue. New Haven: Yale Univ. Obs.

Houk N., 1982, Univ. of Michigan Catalogue of Two-
Dimensional Spectral Types for the HD Stars. Ann Arbor: Univ. Michigan, Vol. 3

Houk N., Smith-Moore M., 1988, Univ. of Michigan Catalogue of Two-Dimensional Spectral Types for the HD Stars. Ann Arbor: Univ. Michigan, Vol. 4

Hynek J.A., 1938, Perkins Obs. Contrib. No. 10, 1, 185

Jaschek C., Jaschek M., 1987, The Classification of Stars. Cambridge University Press

Jaschek M., Andrillat Y., Jaschek C., 1991, A\&A 250, 127

Keenan P.C., Keller G., 1953, ApJ 117, 421

Keenan P.C., McNeil R.C., 1989, ApJS 71, 245

Kuhi L.V., 1963, PASP 75, 448

Levato H., Abt H.A., 1978, PASP 90, 429

Malanushenko V.P., Polosukchina N.S., Weiss W.W., 1994, A\&AS 105, 125

Malaroda S., 1975, AJ 80, 637

Markowitz A.H., 1969, A Study of Stars exhibiting Composite Spectra, Ph.D thesis, Ohio State Univ.

Mason B.D., McAlister H.A., Hartkopf W.I., Griffin R.F., Griffin R.E.M., 1997, AJ 114, 1607

Moore J.H., Paddock G.F., 1950, ApJ 112, 48

Morgan W.W., Keenan P.C., 1973, ARA\&A 11, 29

Olsen E.H., 1979, A\&AS 37, 369

Olsen E.H., 1980, A\&AS 39, 205

Parsons S.B., 1982, PASP 94, 642

Parsons S.B., Ake T.B., Hopkins J.L., 1985, PASP 97, 725

Roman N.G., 1949, ApJ 110, 205

Roman N.G., 1955, ApJS 2, 195

Sato K., Kuji S., 1990, A\&AS 85, 1069

Slettebak A., 1955, ApJ 121, 653

Slettebak A., 1982, ApJS 50, 55

Stephenson C.B., 1960, AJ 65, 60

Stephenson C.B., Nassau J.J., 1961, ApJ 134, 222

Stephenson C.B., Sanwal N.B., 1969, AJ 74, 689

Stickland D.J., 1988, A Decade of UV Astronomy with IUE, Proc. Celebratory Symposium, ESA sp-281, 2, 27 The Hipparcos and Tycho Catalogues, ESA, Noordwijk, ESA SP 1200, 1997

Underhill A., 1954, AJ 59, 332

Walker E.N., 1966, The Observatory 86, 154

Wellmann P., 1951, Astron. Nachr. 279, 257

Wilson R.E., Joy A.H., 1952, ApJ 115, 157

Wright K.O., 1970, Vistas Astron. 12, 147 